\title{
Object Tracking by Filtering Neuron Network
}

\author{
Member Osamu Yoshie \\ (Science University of Tokyo) \\ Non-member Ryuichi Hoshi \\ (Science University of Tokyo) \\ Non-member Junichi Aoki \\ (Canon Sales Co., Inc.) \\ Non-member Shigeki Yamagata \\ $(N$ E C)
}

Object tracking simulation using neural network is described. Since control value for tracking should be determined in accordance with past state, such model is needed that can process time series. So a neuron model called "filtering neuron" is introduced. The idea is quite simple and natural: neuron with filtering synapse weight would deal with time-variant patterns well. Though this is not a new idea at all, back propagation algorithm for the network composed of filtering neurons is not introduced yet. The tracking simulation demonstrates the performance of the model, and smart tracking strategy is realized.

Key words : Object tracking, Neural network, Back propagation algorithm, Time series processing, Filtering

\section{Introduction}

Realizing automatic object tracking with computer is an important problem in the field such as radar system and robot vision. It is strongly desirable that the object should be tracked in a smart manner if the motion can be predicted or constrained to some extent. Conventionally numerical model approach, e.g. $\alpha \beta$ filter method ${ }^{(1)}$, has been a common way to this problem. This kind of way, however, is not appropriate for reflecting explicit strategy to the model therefore makes tracking far from intelligent one.

In contrast with it, connectionist approach, which has been applied to various kinds of problems and realized to be efficient, may provide flexible and intelligent way of tracking because of its learning ability as well as generalizing ability. It is expected that neural network extracts essential strategy from tracking example given as training data. Generally control value for tracking should be determined in accordance with past state. From this point of view a model which is able to deal with input patterns as time series is needed. The state of each cell is modeled only through spatial sum mation in hierarchical network with back propagation algorithm, which is the most common recently. The effects of time-variance ignored. This leads to the fact that for processing time series with back propagation model, either :

(1) Putting input data into buffer and giving them to input layer of the network as a batch, or (2) storing the past information in the network should be done.

Each has its own shortage. Based upon (1), the network will become large. In (2) approach, the way of connecting cells is well considered, and recurrent network model, which has feedback loops between layers, is well known. This model, however, needs much amount of memories and calculations, and its optimizing algorithm includes approximation ${ }^{(2)}$.

So we begin with advocating and introducing new neuron model, called "filtering neuron", then describe the detail of tracking simulation using this model. Filtering neuron has an ability of storing past information, and this mechanism is realized by convolving input data to each cell to infinite impulse response signal. That is, we adopt filtering function instead of constant synapse weight. This itself is not a new idea at al1 ${ }^{(3)}$, but it is a significant result to show that error back propagation is enabled without approximation in hierarchical network of filtering neurons.

We can examine the performance of simulation via $\mathrm{CG}$ system developed on HP workstation. Almost all the works, from constructing network to learning, are able to be done under interactive graphical environment.

What kind of information we can get and how preprocessing should be done to get input data to network are important problems. Since we try to make it clear whether our model is available for object tracking or not, we do not focus on preprocessing. Object motion is 


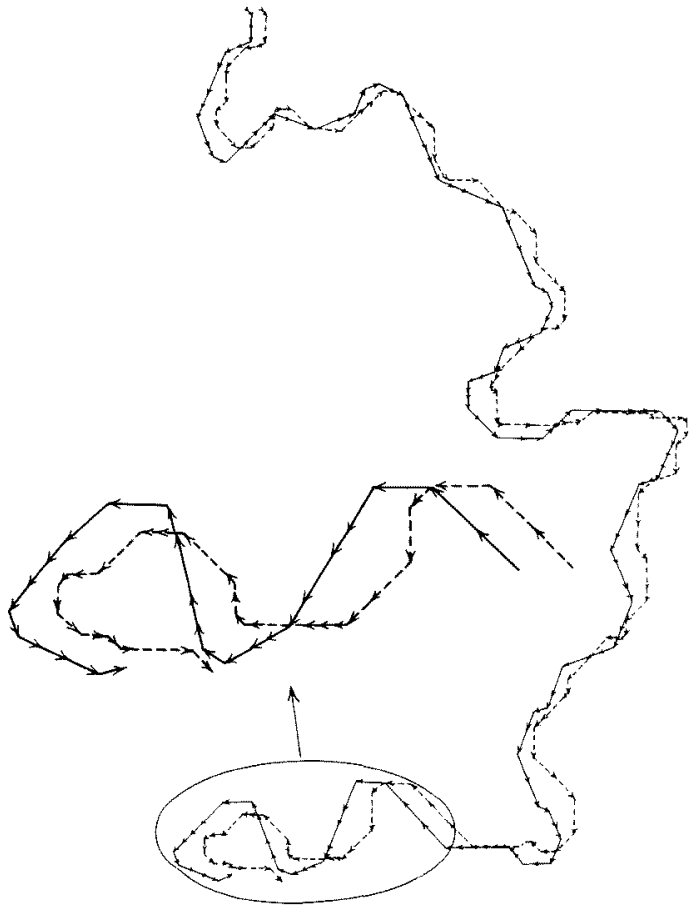

(a) $\alpha \beta$ method

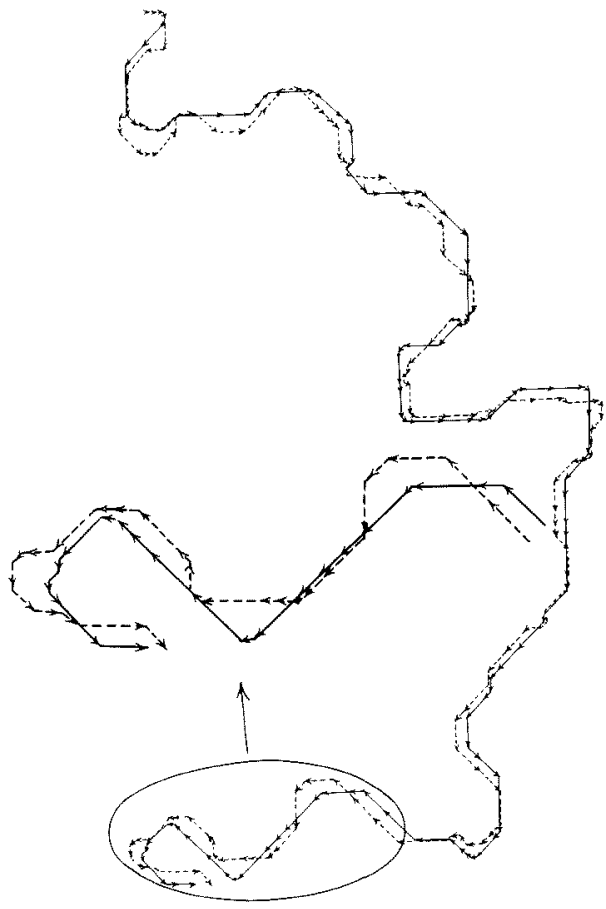

(b) proposed method.

Dotted lines denote the locusses of the tracked object, though both lines are completely same. In both methods the tracker moves as soon as the object escapes.

Fig. 1. Tracking results by different methods.

regarded as to be represented as the sequence of quantized vectors.

\section{Object tracking and neural network}

There are many researches or studies on object tracking. Numerical model approach is quite common and $\alpha \beta$ filter method ${ }^{(1)}$ is the representative one. As shown in Fig. 1, however, the performance of this model is not satisfactory. Generally this kind of model involves troublesome trade off problem: the model could output completely same patterns as input by setting parameters to certain value. Since too much sensibility is not desirable for practical use, we make the model insensitive intentionally. In this approach it is difficult to reflect intelligent strategy to the model.

Rule based approach is also available to embed the strategy of tracking, that is, expressing the strategy for specific pattern in a rule base. It goes without saying that skillful acquisition of efficient rules is necessary. This task is not so easy. Consider to cope with various kinds of motions that appear in practical tracking and slightly differ from those of learning. Contrast with this approach, connectionist one is a "direct" way in the sense that we only have to give raw data for learning, without extracting neat rules from data. Even if a similar pattern to learning data appears, it would be processed well by generalizing ability of neural network.

Mainly from these points of view, we adopt neural network approach.

\section{Model}

In this chapter a neural network model used for tracking problem is described. To realize the strategy depending on the past state, the model should have the ability of processing time series information. It is even harmful to make the model complicated for coping with static patterns. For time-variant patterns, however, it is rather natural to consider the affects with respect to time for each cell. This is done by adopting linear filter instead of constant value for synapse weight. That is, output of a neuron in preceding layer is convolved to impulse response of the filter, and is made contribution to the inner state of neuron. To store past information, it is necessary that the impulse response has long transient 
time to some extent. Therefore IIR (Infinite Impulse Response) filter is more suitable for weighting filter than FIR (Finite Impulse Response) filter, since the number of network parameters would become less by using IIR filter.

In the explanation below, following notations are used :

$\theta_{i}^{k}:$ threshold value of the $i$-th cell in the $k$-th layer $n_{k}$ : the number of cells in the $k$-th layer

$W_{i, j}^{k, k+1}(z)$ : weighting filter between the $i$-th cell in the $k$-th layer and the $j$-th cell in the $(k+1)$-th layer

$$
=\frac{b_{i, j, 0}^{k, k+1}+b_{i, j, 1}^{k, k+1} z^{-1}+\cdots+b_{i, k, m}^{k, k+1} z^{-m}}{1-a_{i, j, 1}^{k, k+1} z^{-1}-\cdots-a_{i, j, n}^{k, k+1} z^{-n}}=\frac{B_{i, j}^{k, k+1}(z)}{A_{i, j}^{k, k+1}(z)}
$$

where $a$ 's and $b$ 's are coefficients of the pulse transfer function. The orders of numerator and denominator are $m$ and $n$, respectively.

$R_{i}^{k}(z)$ : feed back coefficient of the $i$-th cell in the $k$ -th layer

$$
=r_{i 1}^{k}+r_{i 2}^{k} z^{-1}+\cdots+r_{i n}^{k} z^{-n+1}
$$

$x_{i}^{k}(t)$ : inner state of the $i$-th cell in the $k$-th layer at time $t$

$O_{i}^{k}(t)$ : output of the $i$-th cell in the $k$-th layer at time $t$

$y_{i}^{M}(t)$ : teaching signal for the $i$-th cell in the $M$-th layer (output layer) at time $t$

$f(x)$ : I/O function

$E^{M}:$ mean square error of the output layer

$$
=\frac{1}{2} \sum_{i=1}^{n_{M}}\left\{y_{i}^{M}(t)-o_{i}^{M}(t)\right\}^{2}
$$

$\delta_{i}^{k}(t)$ : error of the $i$-th cell in the $k$-th layer at time $t$

$$
=\frac{\partial E^{M}}{\partial x_{i}^{k}(t)}
$$

We call the model "filtering neuron" whose synapse weights are pulse transfer functions of IIR filter, not constant. In this model the output calculation of the $j$-th cell in the $(k+1)$-th layer is:

$$
\begin{aligned}
& x_{j}^{k+1}(t)=\sum_{i=1}^{n_{k}} u_{i, j}^{k, k+1}(t)-\theta_{j}^{k+1} \\
& u_{i, j}^{k, k+1}(t)=W_{i, j}^{k, k+1}(z) o_{i}^{k}(t) \\
& o_{j}^{k+1}(t)=f\left\{x_{j}^{k+1}(t)\right\}
\end{aligned}
$$

Now we derive error back propagation and parameter innovation procedures. First of all, parameter innovation process is based upon following manner:

$$
\begin{aligned}
& a_{i, j, s}^{h, k+1} \leftarrow a_{i, j, s}^{k, k+1}+\eta_{1} \frac{\partial E^{M}}{\partial a_{t, j, s}^{k, k+1}} \\
& b_{i, j, s}^{k, k+1} \leftarrow b_{i, j, s}^{k, k+1}+\eta_{2} \frac{\partial E^{M}}{\partial b_{i, j, s}^{k, k+1}}
\end{aligned}
$$

where

$$
\frac{\partial E^{M}}{\partial a_{i, j, s}^{k, k+1}}=\frac{\partial E^{M}}{\partial x_{j}^{k+1}(t)} \frac{\partial x_{j}^{k+1(t)}}{\partial a_{i j s}^{k+k+1}}
$$

$$
\begin{aligned}
& =\delta_{j}^{k+1}(t) u_{i, j}^{k, k+1}(t-s) \\
\frac{\partial E^{M}}{\partial b_{i j s}^{k+k+1}} & =\frac{\partial E^{M}}{\partial x_{j}^{k+1}(t)} \frac{\partial x_{j}^{k+1}(t)}{\partial b_{i, j}^{k, k+1}} \\
& =\delta_{j}^{k+1}(t) o_{i}^{k}(t-s)
\end{aligned}
$$

So the increments of the parameters are given as follows:

$$
\begin{aligned}
& \Delta a_{i j s}^{k, k+1}=\eta_{1} \delta_{j}^{k+1}(t) u_{i j}^{k, k+1}(t-s) \\
& \Delta b_{i j s}^{h, k+1}=\eta_{2} \delta_{j}^{k+1}(t) o_{i}^{k}(t-s)
\end{aligned}
$$

In the above expression, if $k \neq M$ then $\delta_{j}^{k+1}(t)$ can be calculated by the error back propagation below.

$$
\begin{aligned}
\delta_{i}^{k}(t) & =\frac{\partial E^{M}}{\partial x_{i}^{k}(t)} \\
= & \sum_{j=1}^{n_{k+1}} \frac{\partial E^{M}}{\partial x_{j}^{k+1}(t)} \frac{\partial x_{j}^{k+1}(t)}{\partial O_{i}^{k}(t)} \frac{\partial o_{i}^{k}(t)}{\partial x_{i}^{k}(t)} \\
= & f^{\prime}\left\{x_{i}^{k}(t)\right\} \sum_{j=1}^{n_{k+1}} W_{i, j}^{k, k+1}(z) \delta_{j}^{k+1}(t) \\
= & f^{\prime}\left\{x_{i}^{k}(t)\right\} \sum_{j=1}^{n_{k+1}} \zeta_{i, j}^{k, k+1}(t) \\
\zeta_{2, i}^{k, k+1}(t) & \equiv W_{i j}^{k, k+1}(z) \delta_{j}^{k+1}(t) \\
& =\sum_{i=0}^{m} b_{i, j, i}^{h, k+1} \delta_{j}^{k+1}(t-l)+\sum_{i=1}^{n} a_{i, j, l}^{k, k+1} \xi_{i, j}^{k, k+1}(t-l)
\end{aligned}
$$

If $k=M$ (in the case of output layer) then

$$
\delta_{i}^{M}(t)=\left\{y_{i}^{M}(t)-o_{i}^{M}(t)\right\} f^{\prime}\left\{x_{i}^{M}(t)\right\}
$$

This model, however, is not suitable for practical use too much memories and computation are needed for implementation. So all the AR parts of weighting filters are made common per each neuron. Then we get

$$
A_{i, j}^{k, k+1}(z)=1-z^{-1} R_{j}^{k+1}(z) \text {, for all } 1 \leq i \leq n_{k}
$$

about the weights between $k$-th and $(k+1)$-th layer.

Therefore the output calculation of the $j$-th cell in the $(k+1)$-th layer at time $t$ is as follows:

$$
\begin{aligned}
x_{j}^{k+1}(t)= & \sum_{i=1}^{n_{k}} B_{i, j}^{k, k+1}(z) o_{i}^{k}+ \\
& +R_{j}^{k+1}(z) x_{j}^{k+1}(t-1)-\theta_{j}^{k+1} \\
o_{j}^{k+1}(t)= & f\left\{x_{j}^{k+1}(t)\right\}
\end{aligned}
$$

This model, filtering neuron, is shown in Fig. 2. Note Fig. 2 indicates that ordinary weight is replaced with FIR filter and the neuron has a feedback loop of its own state. The error back propagation and parameter innovation procedures are summarized below:

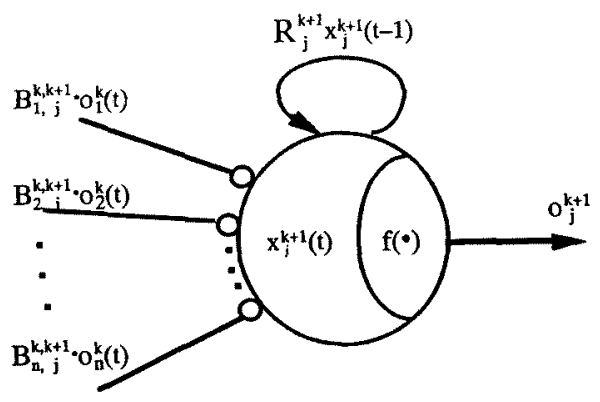

Fig. 2. Filtering neuron model.

T. IEE Japan, Vol. 114-C, No.9, '94 
Error back propagation :

$$
\begin{aligned}
& k=(M-1) \sim 1 \\
& \delta_{i}^{k}(t)=f^{\prime}\left\{x_{i}^{k+1}(t)\right\} \sum_{j=1}^{n_{k+1}} \zeta_{i, j}^{k, k+1}(t) \\
& \zeta_{i, j}^{k, k+1}(t)=B_{i, j}^{k, k+1}(z) \delta_{j}^{k+1}(t)+R_{j}^{k+1}(z) \zeta_{i j}^{k, k+1}(t-1)
\end{aligned}
$$

Parameter innovation:

$$
\begin{aligned}
& K=(M-1) \sim 1 \\
& \Delta r_{i, s}^{k+1}(t)=\eta_{1} \delta_{j}^{k+1}(t) u_{j}^{k+1}(t-s) \\
& +\alpha_{1} \Delta r_{j}^{k+1}(t-1) \quad(s=1 \sim n) \\
& \Delta b_{i, j, s}^{k, k+1}(t)=\eta_{2} \delta_{j}^{k+1}(t) O_{i}^{k}(t-s) \\
& +\alpha_{2} \Delta b_{i, j, s}^{k, k+1}(t-1) \quad(s=0 \sim m) \\
& \Delta \theta_{j}^{k+1}(t)=\eta_{2} \delta_{i}^{k+1}(t)+\alpha_{2} \Delta \theta_{j}^{k+1}(t-1)
\end{aligned}
$$

\section{Object tracking simulation}

Now we describe the simulation using the model mentioned above. The aim is to trace, not to catch, the object $A$ by the scope whose center is $B$. As we consider the center of mass of the object, both $A$ and $B$ are regarded as points. The movement of $A$, which is necessary as input to neural network, is quantized to one of the fundamental vectors by appropriate preprocessing. The output of neural network, control vector of $B$, is also quantized to fundamental vector. The fundamental vectors are shown in Fig. 3. Quantization is done about both direction and distance. Fig. 3 also shows the correspondence of binary codes with these vectors and a sequence of vectors for a motion.

\subsection{Network architecture}

The neural network for simulation, as shown in Fig. 4, is composed of three subnetworks. These three are of ordinary layered type except that filtering neurons are arranged to three layers, and are called NEURO 1, NEURO 2 and NEURO 3, respectively. The output layers of NEURO 2 and NEURO 3 are connected to the input layer of NEURO 1. Each subnetwork has three layers and the numbers of cells are:

\section{NEURO 1 NEURO 2 NEURO 3}

$\begin{array}{lrll}\text { input layer } & 18 & 3 & 3 \\ \text { hidden layer } & 12 & 8 & 8 \\ \text { output layer } & 6 & 3 & 3\end{array}$

where the numbers of hidden layers are determined empirically. The input 3 bits to NEURO 2 and NEURO 3 represent the direction and the magnitude of $A$ 's movement, respectively. NEURO 2 and NEURO 3 process these data as time series and pass 3 bits output to NEURO 1. The 3 bits pattern from NEURO 2 stands for the shape of $A$ 's movement, and that of NEURO 3 represents the trend of $A$ 's velocity. Both output pat. terns are determined from the information of time interval $[t-2, t]$ where $t$ is current time. It may not be direction

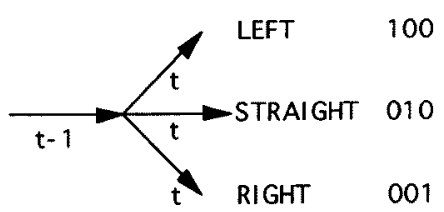

distance

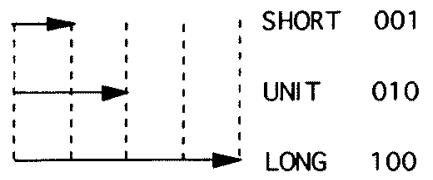

Fig. 3. Fundamental vectors.

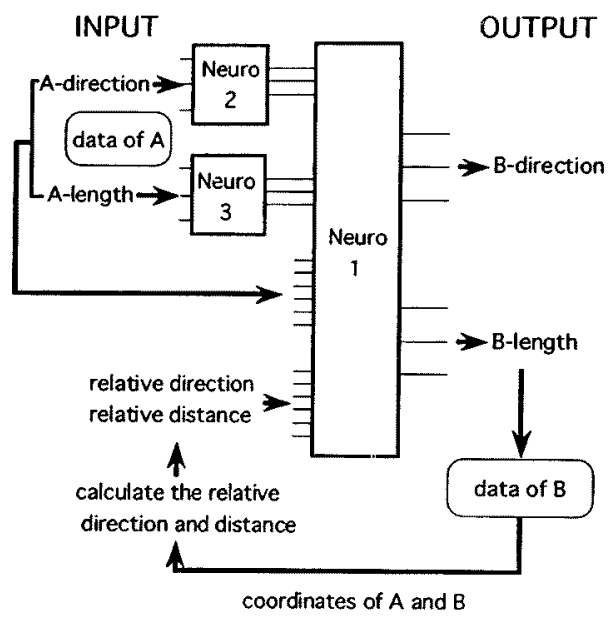

Fig. 4. Network architecture.

necessary to apply connectionist approach to these preprocessing modules. We adopt this approach only because of the consistency of total neural network processing. The input pattern to NEURO 1 consists of 18 bits: 6 bits from NEURO 2 and NEURO 3, same 6 bits as input to NEURO 2 and NEURO 3, 3 bits for relative direction between $A$ and $B$ and 3 bits for relative distance. 3 bits for direction and other 3 bits for magnitude corresponding to vector of $B$, which are necessary for tracking, are acquired as output of NEURO 1.

\subsection{Learning}

Learning process is divided into two stages: NEURO 2 and NEURO 3 are trained independently so that they can recognize shape and amplitude of motion patterns, 


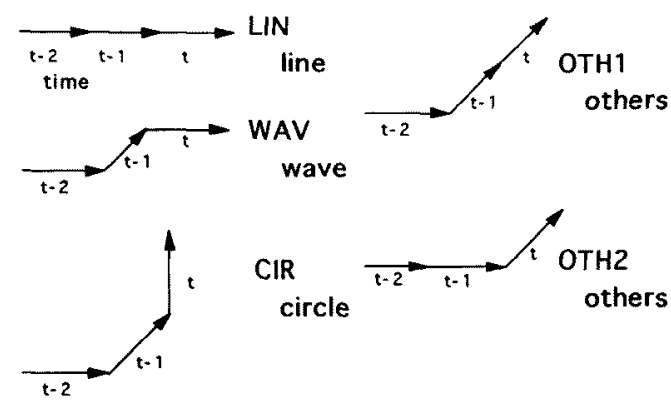

Fig. 5. The shapes that NEURO 2 can recognize.
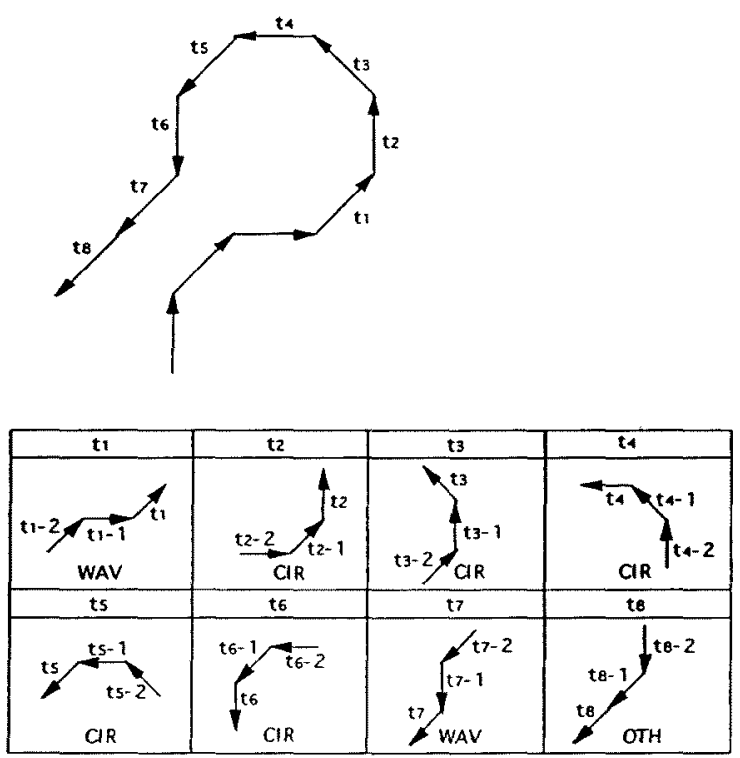

Fig. 6. An example of learning for NEURO 2

respectively, then total network is trained to output the control value of $B$, the center of scope. Since all subnetworks are composed of same type of filtering neur. ons, the ways of giving input signal and teaching signal are similar. That is, not that buffered information is given to network at the same time, but that a pair of (input signal, teaching signal) is given at each time.

The number of shapes that NEURO 2 can recognize is 9 , including symmetric patterns of what we can see in Fig. 5. An example of learning is given in Fig. 6. Note that input signal is only last one vector of the pattern, though teaching signal is the shape of latest three vectors. For the learning of NEURO 3 teaching signal is given in such a way that it categorizes the patterns composed of latest three vectors, from SHT-SHT-SHT to LONG-LONG-LONG, into three classes, LNG, MID and SHT. The learning of two subnetworks is successfully finished in $10^{2}$ order iterations.

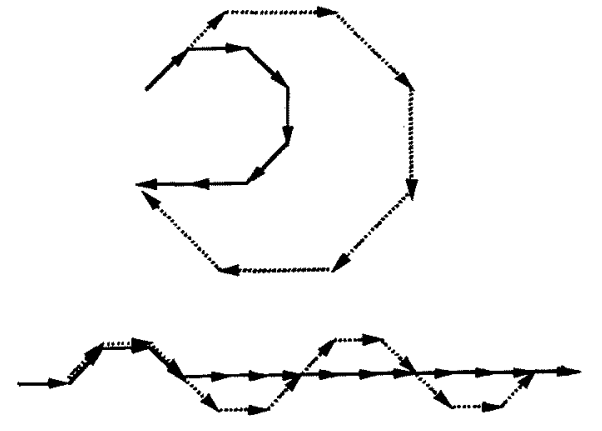

Dotted line denotes motion patterns of the tracked object.

Fig. 7. Two examples of tracking strategy.

Essential learning for tracking is done for interconnected network, where NEURO 2 and NEURO 3 have been trained in above manner. Using mouse, human operator actually wakes tracks for the sequence of patterns that compuer generated from next to next. One sequence is composed of about 500 vectors ( 512 vectors at most), and four sequences are tracked for data acquisition. By giving these as training data to the network, it is expected that his strategy is strongly reflected to the tracking. Fig. 7 demonstrates the examples of tracking strategy. $10^{4}$ order iterations are needed for the convergence of learning, where learning parameters are:

$$
\begin{array}{lll}
\text { learning coefficients } & \alpha_{1}=0.9, & \alpha_{2}=0.1 \\
\text { momentums } & \eta_{1}=0.1, & \eta_{2}=0.01
\end{array}
$$

\subsection{Results and considerations}

Fig. 1(b) shows that trained network tracks the pattern appropriately which computer geneates randomly. In this pattern generation process, the probabilities to which appearance of fundamental vectors obey are given. Especially when the motion pattern can be predicted, good maneuver-following is realized, which cannot be expected by $\alpha \beta$ filter method shown in Fig. 1 (a). In Fig. 1(a) tracker sometimes "overshoots" when the object changes its direction, while rarely observed in Fig. 1 (b). Our tracking simulator can also display the result by real time computer graphics, though Fig. 1 only shows the locus of motion.

Table 1 shows the synapse filters coefficients acquired by learning. Though the filters are of IIR, equivalent FIR filters can be got by dividing the numerators by denominators. In Table 1 are there coefficients of the FIR filters. The filters on which we are focusing now are the ones between input layer and hidden layer. What is important here is that the coefficients corresponding to higher orders than $z^{-2}$ are rather small. Since the order 
Table1. Coefficients of synapse FIR filters acquired by learning.

\begin{tabular}{c|r|r|r|r}
\hline \multirow{2}{*}{$\begin{array}{c}\text { cell in the } \\
\text { hidden layer }\end{array}$} & \multicolumn{4}{|c}{ FIR coefficients } \\
\cline { 2 - 5 } & \multicolumn{1}{|c|}{$z^{-0}$} & \multicolumn{1}{c}{$z^{-1}$} & \multicolumn{1}{c}{$z^{-2}$} & \multicolumn{1}{c}{$z^{-3}$} \\
\hline 1 & 2.27 & -1.12 & 2.28 & -0.33 \\
\hline 2 & 3.85 & -4.05 & 0.40 & -0.04 \\
\hline 3 & -3.25 & 4.44 & 1.20 & -0.18 \\
\hline 4 & -2.93 & 2.53 & 0.14 & 0.02 \\
\hline 5 & -1.24 & -0.84 & 0.73 & 0.03 \\
\hline 6 & 4.83 & 5.26 & 2.09 & 0.31 \\
\hline 7 & -2.88 & 3.89 & -0.37 & 0.34 \\
\hline 8 & 3.61 & 3.59 & -0.52 & -0.62 \\
\hline
\end{tabular}

This table shows equivalent FIR filters between a cell in the input layer and 8 cells in the hidden layer.

of filter between input layer and hidden layer is concerning the way of capturing input data, our network found the answer of next question: how many data in the past should the network take into account to recognize input pattern? Namely, the network learned the way of data segmentation. The result is quite reasonable, because patterns we gave as teaching data are composed of 3 vectors each.

\section{Conclusion}

We adopt neural network approach for tracking problem. To realize smart maneuver-following, we introduced new neuron model called "filtering neuron". This is the model which realizes time series processing by state feedback and cutting off unnecessary past input patterns. That is, inner state dynamics is taken into account. This is quite different from conventional models, such as Jordan sequential network ${ }^{(3)}$ and Elman's simple recurrent network ${ }^{(4)}$ as well as ordinary recurent network. Though the networks which involve feedback loops cannoot help spending much calculation and memories more or less, our model can. Further, no approximation is needed to acquire error back propagation for whole network. The model can deal with dynamic patterns directly as well static patterns. The model has an ability to decide how many data in the past should be taken into account to recognize input Pattern. This fact indicates that the model can be applied to some data segmentation problems in the field such as acoustic signal processing.

(Manuscript received Nov. 1, '93

revised Apr. 18, '94)

\section{References}

(1) T. R. Benedict \& G. W. Bordner: "Synthesis of an optimal se of rader track-while-scan smoothing equations", IRE Trans. Automatic Control, AC-7, 27 (1962)

(2) D. E. Rumelhurt, J.L. McClelland \& the PDP Research Group : Parallel distributed processing, MIT Press

(3) M. I. Jordan: "Attractor dynamics and parallelism in a con nectionist sequential machine", Proc. Eighth Annual Conference of the Cognitive Science Society

(4) J. L. Elman: "Finding structure in time", CRL Technical Report 8801, Center for Research in Language, University of california, San Diego.

（5）甘利俊一：神経回路網の数理, 産業図量

(6) A. J. Kanyuck: "Transient response of tracking filters with randomly interrupted data", IEEE Trans. Aerospace \& El ctronic Systems, AES-6, No. 3, 313 (1970)

Osamu Yoshie

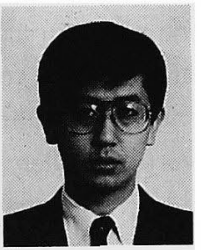

Ryuichi Hoshi (Non-member) He is now a graduate student

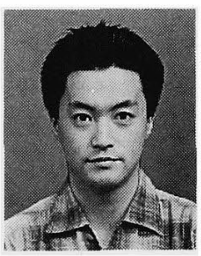

Junichi Aoki (Non-member) He graduated from Science

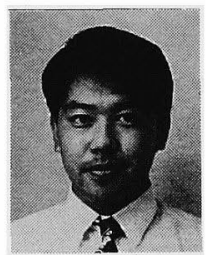
of Science University of Tokyo. He is studying about neural networks, database systems and multi-media.

(Member) He is a lecturer in the department of Applied Physics, Faculty of Science, Science University of Tokyo. He got Dr. Eng. degree from Waseda University. His main interests are image understanding, machine learning and human-computer-communications.

University of Tokyo in 1993. And he works at Canon Sales Inc.
Shigeki Yamagata (Non-member) He works at Guidance \&

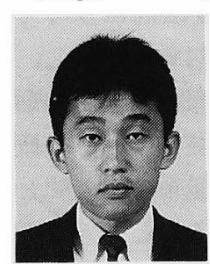

